\title{
Efecto de la longitud de onda en la respuesta dinámica de muros de retención embebidos
}

\author{
Effect of the wave length on the dynamic response of embedded retaining walls
}

Fecha de entrega: 2 de diciembre 2014 Fecha de aceptación: 24 de abril 2015

\section{Bogart Méndez ${ }^{1}$ y Diego Rivera ${ }^{2}$}

\author{
${ }^{1}$ Rizzo Associates Chile, Presidente Errázuriz 3724, Las Condes, Santiago, Chile, Bogart.Mendez@rizzoassoc.cl \\ ${ }^{2}$ Rizzo Associates Inc., 500 Penn Center Boulevard, Suite 100, Pittsburgh, PA 15235, USA, Diego.Rivera.@rizzoassoc.com
}

En la respuesta dinámica de muros de retención embebidos existe un potencial efecto de la relación entre la longitud de onda $\lambda$ de la excitación y la altura del muro $H$, o $\lambda / H$, en los empujes sísmicos aplicados sobre la estructura. En este trabajo se investiga este efecto, tomando como antecedente el hecho de que en el caso de una estructura subterránea, los movimientos sísmicos pueden amplificarse al incidir en ella si las longitudes de onda oscilan entre 1 y 4 veces su diámetro. Los análisis utilizan un perfil de suelo granular grueso, similar al encontrado en Santiago, considerando un modelo constitutivo no lineal en conjunto con amortiguamiento histerético. El perfil inicial de velocidades de onda en el suelo corresponde con los valores de $V_{s}$ de los primeros $30 \mathrm{~m}$ de profundidad y representativos de materiales gravosos de la cuenca de Santiago. El modelo numérico contempla fronteras laterales de campo libre, frontera absorbente en la base y elementos interfaz suelo-muro para tomar en cuenta su interacción estática y dinámica. Como excitación se utilizan tanto movimientos armónicos como un registro sísmico en roca característico de los movimientos en Chile, aplicados a la base del modelo. La respuesta en campo libre se compara con los resultados de análisis unidimensionales de propagación de onda, y las presiones dinámicas de tierra se contrastan con los cálculos efectuados mediante el método de Mononobe-Okabe para buscar el rango $\lambda / H$ donde su desempeño es adecuado para el diseño de este tipo de estructuras.

Palabras clave: longitudes de onda, empujes dinámicos, muros de retención
In the dynamic response of embedded retaining walls, there is a potential effect of the wavelength/height ratio $\lambda / H$ over seismic pressures exerted on the wall. This paper addresses this issue, bearing in mind that in the case of underground structures, dynamic motion can be amplified when wavelengths range between 1 and 4 times their diameter. The work herein presented analyzes a coarse granular soil profile, similar to that found in Santiago. A non-linear constitutive model is used coupled with hysteretic damping. The initial shear wave velocity profile corresponds to $V_{s}$ in the first 30 $m$ depth with values representative of gravelly material of Santiago basin. The numerical model developed uses free field lateral boundaries (compatible with non-linear soil behavior), absorbing boundary at the bottom and interface elements between the soil and the wall to account for static and dynamic interaction. Input motion corresponds to both harmonic time series and a rock motion characteristic of Chile earthquakes. The motions were applied to the model's base. Free field model response was compared to one dimensional wave propagation analysis and dynamic earth pressures were compared to Mononobe-Okabe calculations to investigate the $\lambda / H$ range were its performance is adequate for the design of these type of structures.

Keywords: wave length, dynamic soil pressures, retaining walls

\section{Introducción}

Se presenta una investigación numérica sobre el efecto de la longitud de onda de la excitación $\lambda$, en las presiones sísmicas de un muro de retención embebido. El término muro de retención embebido se refiere a una estructura que se coloca para retener una excavación. Un muro de retención convencional, ya sea del tipo masivo o cantiléver, se considera como aquél que se forma mediante material de relleno. Los resultados se presentan en términos de la relación $\lambda / H$, donde $H$ es la altura del muro que retiene a 


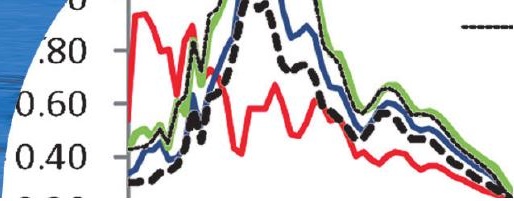

la excavación. Para generar diversas longitudes de onda se utilizan funciones armónicas de distintas frecuencias, todas con el mismo nivel de aceleración máxima e igual duración. Finalmente se realiza un análisis con un registro sísmico con un contenido de frecuencias que genera diversas relaciones $\lambda / H$ simultáneamente.

\section{Calibración del modelo constitutivo bajo carga estática}

Para simular el comportamiento del material granular se utilizó el modelo constitutivo no lineal de Mohr-Coulomb. Para las capas más superficiales de material se consideró una variante del modelo Mohr-Coulomb mediante un comportamiento strain-softening para la degradación de la cohesión en función de la deformación plástica. El ángulo de fricción del material y su cohesión se calibraron en función del esfuerzo de confinamiento utilizando resultados experimentales de ensayos triaxiales homotéticos a deformación constante realizados en materiales granulares gruesos (De la Hoz, 2007).

La calibración se llevó a cabo mediante la simulación numérica de los ensayos experimentales, sometiendo una probeta virtual (Figura 1) a etapas de consolidación y corte a deformación controlada. Las simulaciones se ejecutaron en la plataforma numérica FLAC3D (Itasca, 2009). Se programó una función FISH (Itasca, 2009) para monitorear la variación del área transversal de la probeta virtual en su parte superior, y de esta forma conocer la variación del esfuerzo vertical actuante en la muestra. El módulo de Young $E$ durante los ensayos virtuales varía en función del esfuerzo de confinamiento efectivo, de acuerdo con la variación propuesta por De la Hoz (2007) replanteada en términos del esfuerzo normal octaédrico: $E=136080\left(\sigma_{\text {oct }}\right)^{0.48}$, donde $E$ y $\sigma_{\text {oct }}$ están en Pascales. Los ensayos simulados corresponden a los del material P-1 (De la Hoz, 2007), para probetas de $15 \times 30 \mathrm{~cm}$ sometidas a esfuerzos de confinamiento de 100, 200 y $400 \mathrm{kPa}$. Los resultados se presentan en la Figura 2.

Se observa que el modelo de Mohr-Coulomb captura el comportamiento del material granular en forma suficientemente aproximada para utilizarse en los cálculos definitivos. Mediante el uso de modelos constitutivos más elaborados es posible reproducir más de cerca las curvas experimentales. Sin embargo, una calibración detallada

del tipo de modelo constitutivo que reproduzca mejor el comportamiento experimental, está fuera del alcance de este trabajo. En la Tabla 1 se presentan los parámetros utilizados en el modelo constitutivo para generar las curvas mostradas en la Figura 2. La cohesión durante el ensayo de $100 \mathrm{kPa}$ varía con la deformación plástica de corte. La variación se obtuvo a partir de la relación presentada por De la Hoz (2007) entre la cohesión y la deformación axial en una probeta triaxial a gran escala. La relación entre la deformación axial y la deformación plástica de corte se obtuvo mediante simulaciones numéricas de ensayos triaxiales para obtener la relación entre ambos parámetros.

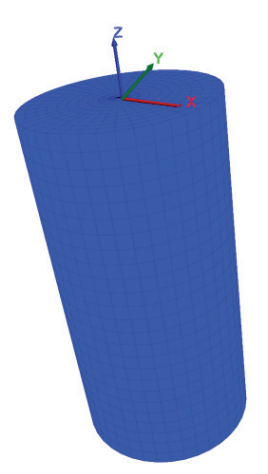

(a)

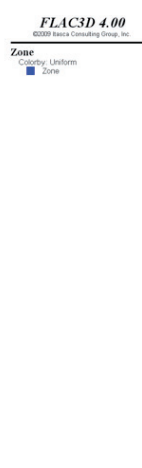

Figura 1: Ensayo triaxial virtual: a) geometría original y b) geometría post ensayo

Tabla 1: Propiedades utilizadas en los ensayos triaxiales numéricos

\begin{tabular}{|l|c|c|c|c|c|}
\hline Ensayo & $\phi{ }^{\circ}$ & $c, \mathrm{kPa}$ & $\psi$ & $\rho, \mathrm{kg} / \mathrm{m}^{3}$ & $v$ \\
\hline $100 \mathrm{kPa}$ & 44 & varía & 3 & 2115 & 0.20 \\
\hline $200 \mathrm{kPa}$ & 42 & 10 & 3 & 2116 & 0.20 \\
\hline $400 \mathrm{kPa}$ & 41 & 21 & 3 & 2161 & 0.20 \\
\hline
\end{tabular}

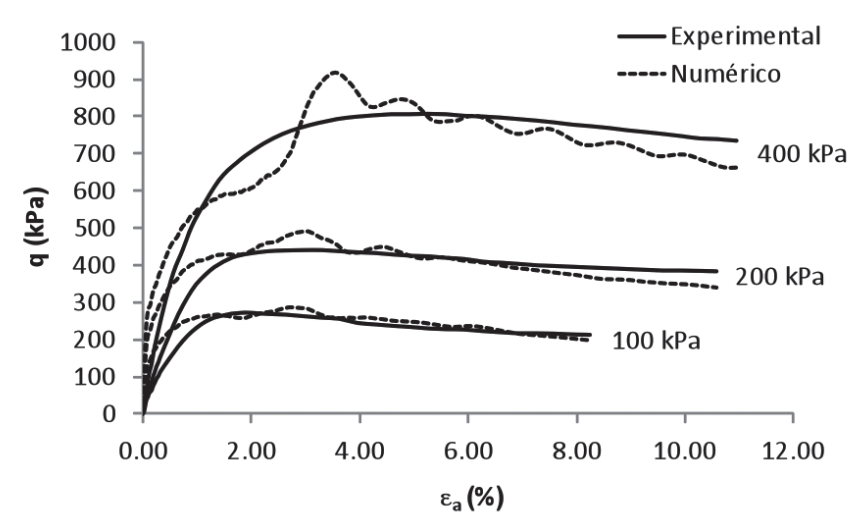

Figura 2: Comparación de resultados experimentales versus numéricos 


\section{Propiedades dinámicas de los materiales}

La variación de la velocidad de propagación de las ondas de corte $V_{\mathrm{s}}$, se modeló a profundidad de acuerdo con valores típicos encontrados en los depósitos gravosos de Santiago. Esta variación se muestra en la Figura 3a, mientras que en la Figura $3 \mathrm{~b}$ se presenta la variación correspondiente del módulo de corte a pequeñas deformaciones $G_{0}$, con la profundidad. Se ha considerado la presencia de la roca basal a la profundidad de $30 \mathrm{~m}$. Las densidades adoptadas son 2200 y $2450 \mathrm{~kg} / \mathrm{m}^{3}$ para la grava y la roca, respectivamente. Se adoptaron curvas de degradación y amortiguamiento para los materiales, variando con la profundidad para tomar en cuenta el nivel de confinamiento en la degradación de las propiedades. Las curvas se presentan en la Figura 3. Como referencia, en las curvas se incluyen los límites de Seed et al. (1986) para materiales granulares. El amortiguamiento se limitó a un valor máximo de $15 \%$ para grandes deformaciones que se encuentran fuera del rango de aplicabilidad del método lineal equivalente.

Sin embargo, se efectuaron análisis para verificar que el nivel de deformaciones de corte inducidas por la carga sísmica, en condiciones de campo libre, estuviese dentro de los límites de aplicación del método lineal equivalente (del orden de $10^{-2} \%$ como máximo). Las curvas de degradación se utilizaron de dos formas distintas: (1) para validar la respuesta unidimensional de campo libre de FLAC3D mediante el método lineal equivalente (a través de comparaciones con SHAKE2000, 2008), y (2) para proporcionar amortiguamiento en la parte elástica de los análisis no lineales. En ambos casos se consideró una deformación de corte efectiva igual al $65 \%$ de la deformación máxima (Idriss y Sun, 1992).

En el caso de los análisis no lineales efectuados en FLAC3D, antes de la fluencia de los materiales se ejecuta el método lineal equivalente para amortiguar los movimientos. Una vez alcanzada la fluencia, las curvas de degradación dejan de utilizarse y la disipación de energía se da de forma natural por efectos de plasticidad del modelo constitutivo.

\section{Respuesta unidimensional de campo libre}

Se verificó la respuesta unidimensional de campo libre obtenida con FLAC3D, considerando las propiedades dinámicas de los materiales antes descritas y aplicando el sismo en la roca basal. Los resultados de FLAC3D se compararon con los obtenidos en SHAKE2000, software estándar para análisis de respuesta de sitio. Para que los resultados fuesen comparables, los análisis en FLAC3D se ejecutaron mediante el método lineal equivalente considerando una deformación de corte efectiva igual al $65 \%$ de la deformación máxima, valor utilizado comúnmente en SHAKE. El sismo empleado en los análisis es el registro en roca de la estación UTFSM de Valparaíso correspondiente al canal 3 del archivo corregido por Boroscheck et al. (2010). La aceleración máxima del registro PGA es de $0.30 \mathrm{~g}$, su duración es de $72 \mathrm{~s}$ y la frecuencia máxima es $25 \mathrm{~Hz}$. El tamaño de los elementos en los análisis de FLAC3D es compatible con el contenido de frecuencias del sismo, de acuerdo con Lysmer y Kuhlemeyer (1969).

Los resultados se presentan en la Figura 4 en términos del perfil de deformaciones de corte máximas, espectros de respuesta y espectro de amplitudes de Fourier. Se observa que la respuesta obtenida con ambos códigos es prácticamente idéntica.
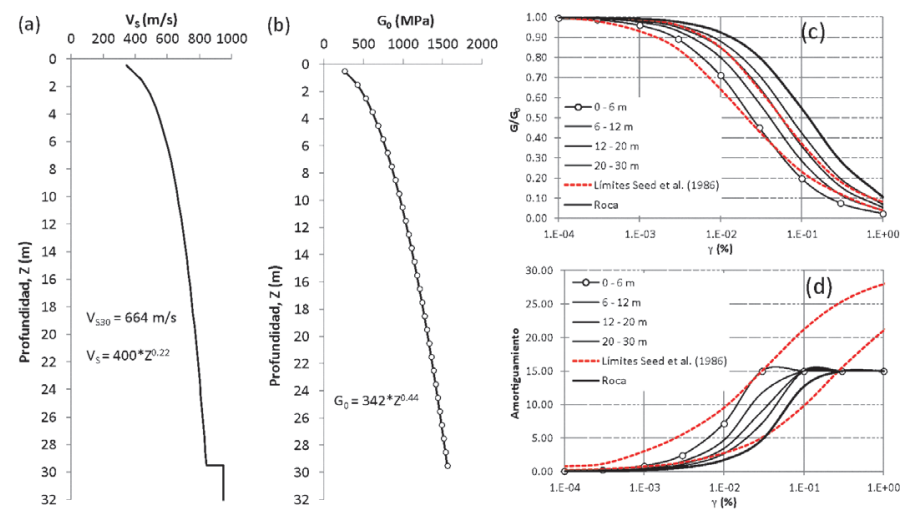

Figura 3: Variación inicial de: a) $V_{\mathrm{s}}$ y b) $G_{0}$ del perfil de suelo considerado, c) y d) curvas de degradación y amortiguamiento adoptadas, respectivamente
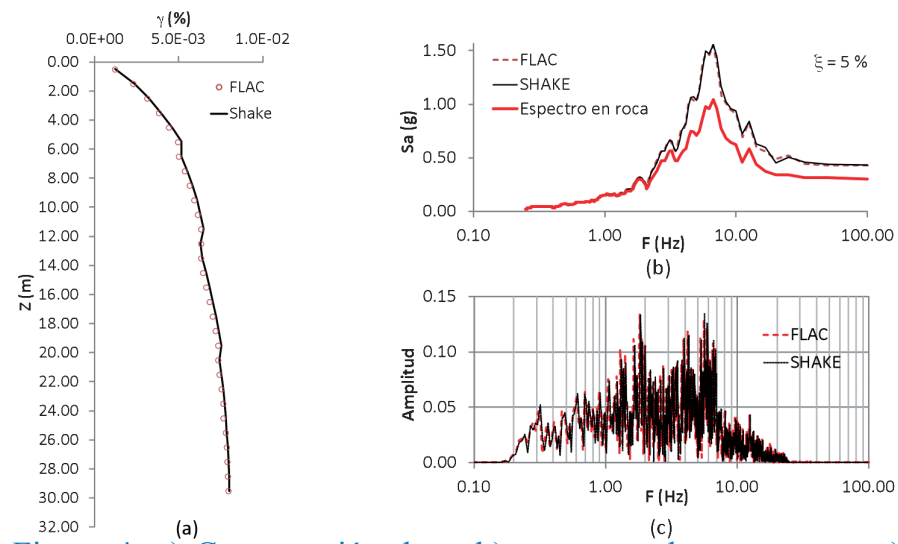

Figura 4: a) Comparación de $\gamma$, b) espectros de respuesta y c) amplitudes de Fourier 


\section{Geometría del muro analizado y modelo numérico}

Para los análisis se adoptó un muro de $5 \mathrm{~m}$ de altura, como se observa en la Figura 5. Los modelos constitutivos de cada material y sus propiedades se muestran en la misma figura. El muro de retención se modeló mediante un elemento elástico lineal tipo liner de doble lado, de tal forma que puede instalarse dentro del suelo y luego realizar la excavación. El contacto entre el suelo y el muro se simula mediante elementos interfaz de comportamiento elastoplástico con un ángulo de fricción de $30^{\circ}$ y cohesión de 0.5 $\mathrm{kPa}$. La rigidez normal y tangencial que unen al elemento liner con la malla principal del modelo se asignaron con base en la rigidez aparente de los elementos de la malla. El tamaño de los elementos en la vecindad del muro es de $0.50 \mathrm{~m}$ y aumenta gradualmente hasta $1.70 \mathrm{~m}$ en torno a la roca. La longitud de la parte enterrada del muro es igual a su altura. Esta es una variable importante en el desarrollo de las presiones sísmicas (Leuzzi et al., 2010), pero su análisis está fuera del alcance de este trabajo.

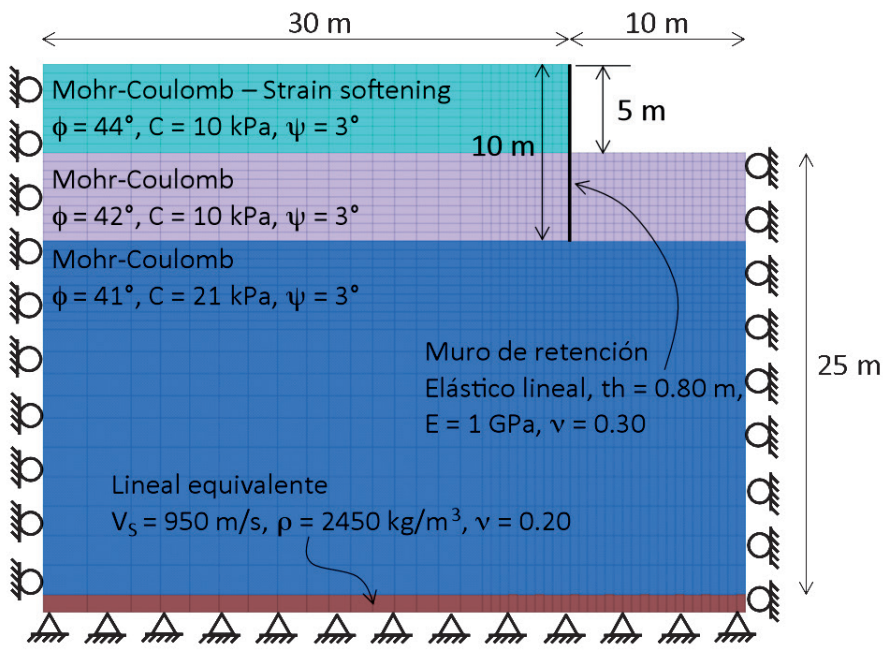

Figura 5: Modelo numérico empleado

\section{Resultados}

\section{Empujes bajo carga estática}

La distribución inicial de esfuerzos geostáticos de campo libre se asignó aplicando un coeficiente de empuje en reposo, $k_{0}=0.32$. La variación del módulo de Young del material se adoptó de acuerdo con la variación calibrada en los ensayos triaxiales virtuales presentados anteriormente. El proceso de análisis bajo carga estática es el siguiente: instalación del estado de esfuerzos geostáticos de campo libre (condición en reposo), activación del elemento liner dentro del suelo y finalmente ejecución de la excavación en cinco etapas de $1 \mathrm{~m}$ de espesor cada una. Durante la excavación el módulo de Young varía en función del esfuerzo de confinamiento.

Los resultados se presentan en la Figura 6a en términos de las presiones de suelo sobre el muro y de desplazamientos relativos a la base del muro, $\mathrm{U}_{\text {rel }}$. Los resultados analíticos de presiones de tierra presentados en la figura se obtuvieron mediante la teoría de Rankine para materiales con cohesión y fricción. El cálculo de la profundidad de la zona de tensión en el suelo coincide entre el método analítico y el numérico. Sin embargo, se observa que los resultados numéricos comienzan a apartarse del caso activo y con la profundidad, cercano a la base del muro, se acercan al valor en reposo. La diferencia se debe a que los resultados numéricos toman en cuenta la rigidez del muro, la cual en este caso, en conjunto con la rigidez misma del suelo, evita que se desarrolle el estado activo por completo. En la Figura $6 \mathrm{~b}$ se muestra la distribución de desplazamientos horizontales del muro, relativos a su base. Se observa que los desplazamientos desarrollados son del orden de $2.5 \mathrm{~mm}$ en la parte superior. Comparado con la altura total del muro $H$, en la Figura $6 c$ se observa que el nivel de desplazamiento no es suficiente para el desarrollo completo del estado activo, congruente con el nivel de esfuerzo desarrollado en el material, el cual se encuentra en un nivel intermedio entre el activo y el de reposo en torno a la base del muro. Tendencias parecidas se han reportado en trabajos similares al reportado en este documento (e.g., Leuzzi et al., 2010; Méndez et al., 2014).

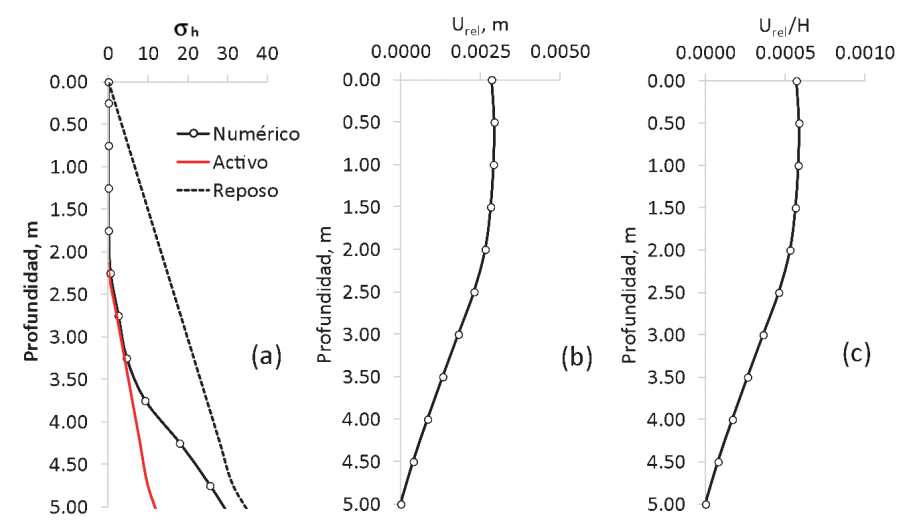

Figura 6: a) Presiones de suelo, b) y c) desplazamientos relativos a la base del muro 


\section{Empujes bajo carga armónica}

Partiendo del estado de esfuerzos estáticos, se aplicaron cargas armónicas de distintas frecuencias $F$, para inducir longitudes de onda específicas para investigar su influencia en la variación de los esfuerzos dinámicos. Las frecuencias de la excitación armónica se eligieron tomando en cuenta la altura del muro y el rango de razones $\lambda / H$ que se deseaba investigar. Si bien las frecuencias son mayores al rango típico de las frecuencias con mayor contenido de energía en los sismos reales, éstas se eligieron para obtener un amplio rango de variación de los cocientes de la frecuencia de la excitación $F$, entre la frecuencia natural del sitio $F_{\text {sitio, }}$ y entre la frecuencia natural del suelo detrás del muro $F_{0}$.

Las señales empleadas en los análisis se presentan en la Tabla 2. La longitud de onda aplicada se calculó con base en el valor de $V_{\mathrm{s} 30}$ del perfil de suelo, $664 \mathrm{~m} / \mathrm{s}: \lambda=V_{\mathrm{s} 30} / F$. Todas las señales tienen la misma amplitud en términos de aceleración $\ddot{U}_{0}$, igual al valor de PGA del registro utilizado como excitación en los análisis de carga sísmica, y la misma duración $T_{\mathrm{d}}=1 \mathrm{~s}$. En la Tabla 2 se presenta también la razón de la frecuencia de la excitación armónica $F$, contra la frecuencia fundamental del perfil de suelo completo por encima de la roca basal $F_{\text {sitio }}$ El valor de esta frecuencia corresponde al obtenido con $V_{\mathrm{s} 30}$, i.e., $F_{\text {sitio }}=V_{\mathrm{s} 30} / 4 H=5.5$ Hz. Como referencia, la frecuencia fundamental del suelo detrás del muro de retención $F_{0}$, es alrededor de $22 \mathrm{~Hz}$ (estimado a partir de la función de transferencia entre la base del muro y la superficie del suelo natural, valor similar al del cálculo elástico 1D).

Tabla 2: Señales armónicas utilizadas

\begin{tabular}{|c|c|c|c|c|c|c|c|c|c|}
\hline Señal & $F, \mathrm{~Hz}$ & $F / F_{\text {sitio }}$ & $F / F_{0}$ & $\lambda, \mathrm{m}$ & $\lambda / H$ & $\ddot{\mathrm{U}}_{0}, \mathrm{~g}$ & $V_{0}, \mathrm{~m} / \mathrm{s}$ & $D_{0}, \mathrm{~m}$ & $T_{\mathrm{d}}, \mathrm{s}$ \\
\hline 1 & 50 & 9.04 & 2.27 & 13 & 3 & 0.30 & 0.0094 & $2.98 \cdot 10^{-5}$ & 1.0 \\
\hline 2 & 25 & 4.52 & 1.14 & 27 & 5 & 0.30 & 0.0187 & $1.19 \cdot 10^{-4}$ & 1.0 \\
\hline 3 & 17 & 3.07 & 0.77 & 39 & 8 & 0.30 & 0.0276 & $2.58 \cdot 10^{-4}$ & 1.0 \\
\hline 4 & 12 & 2.17 & 0.55 & 55 & 11 & 0.30 & 0.0390 & $5.18 \cdot 10^{-4}$ & 1.0 \\
\hline 5 & 10 & 1.81 & 0.45 & 66 & 13 & 0.30 & 0.0468 & $7.45 \cdot 10^{-4}$ & 1.0 \\
\hline
\end{tabular}

Para cada análisis se obtuvo la distribución de esfuerzos dinámicos ejercidos sobre el muro, y con ella se calculó la fuerza de empuje sísmico total (estático + dinámico), $E_{\mathrm{S}}$. El empuje obtenido numéricamente se comparó contra el empuje analítico calculado con el método de MononobeOkabe (M-O), $E_{\mathrm{MO}}$, considerando solamente el ángulo de fricción (sin tomar en cuenta la cohesión). Para los cálcu- los con $\mathrm{M}-\mathrm{O}$ se consideraron dos valores del coeficiente sísmico horizontal utilizados comúnmente en la práctica: $k_{\mathrm{h}}=\mathrm{PGA} / 2$ y $k_{\mathrm{h}}=\mathrm{PGA}$. El coeficiente sísmico vertical se tomó igual a cero. Los resultados se presentan en las Figuras 7 y 8 .

En la Figura 7a se muestra la variación de los empujes normalizados contra las distintas razones $\lambda / H$ para diferentes instantes de aplicación de la carga armónica. La normalización se efectuó considerando $k_{\mathrm{h}}=\mathrm{PGA} / 2$ en el método M-O. Se observa que el valor de los empujes se estabiliza hacia el final de la aplicación de la carga. Los valores medios de los empujes normalizados, durante la aplicación de la carga, se muestran en la Figura $7 \mathrm{~b}$ para los casos $k_{\mathrm{h}}=\mathrm{PGA} / 2$ y $k_{\mathrm{h}}=\mathrm{PGA}$ de M-O. En la Figura $7 \mathrm{~b}$ se muestran las razones de frecuencia $F_{\text {sitio }}$ y $F / F_{0}$, donde los resultados indican una tendencia de aumento de los empujes sísmicos con el incremento de la razón $\lambda H$. Sin embargo, dado que todas las razones $\lambda / H$ poseen un cociente $F_{\text {sitio }}$ distinto, la variación observada en los empujes sísmicos normalizados probablemente esté más influenciada por la relación $F_{\text {sitio }}$ que por la razón $\lambda H$. Se observa que conforme el cociente $F_{\text {sitio }}$ se acerca a la unidad, se produce un claro aumento de los empujes, hasta que comienza a decrecer para el valor $F_{\text {sitio }}=1.81$, debido tal vez a efectos no lineales en la respuesta del suelo. Este tipo de efectos de disipación de energía por plasticidad en el material se ha observado en estudios similares (e.g., Leuzzi et al., 2010), donde las presiones dinámicas sobre elementos de retención flexibles disminuyen por efectos no lineales.
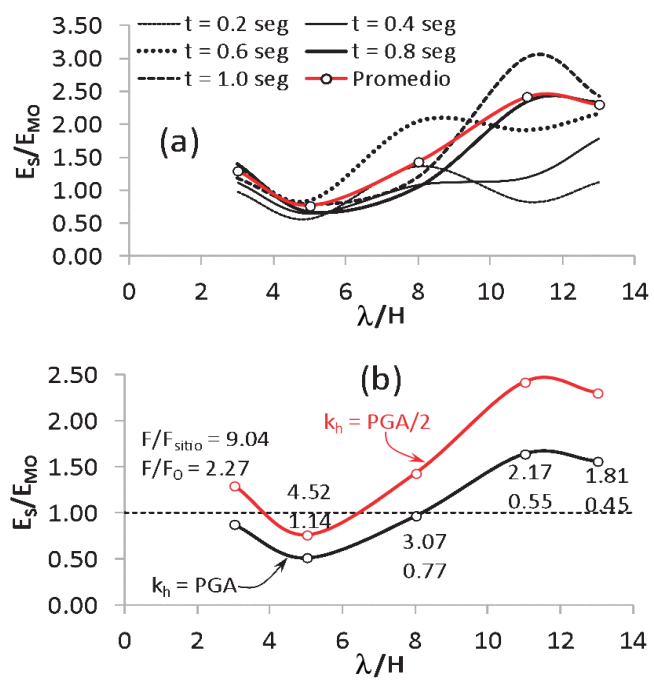

Figura 7: a) Empujes normalizados y b) curva promedio, caso de carga harmónica 
Es interesante observar que para el caso $k_{\mathrm{h}}=\mathrm{PGA} / 2$, todos los empujes calculados son mayores que el valor entregado por el método de Mononobe-Okabe (salvo el caso para $\lambda / H=5$ ). Por otro lado, cuando se considera $k_{\mathrm{h}}$ $=$ PGA, los resultados con M-O sobreestiman los cálculos numéricos, excepto para los casos con $\lambda / H>8$. Sin embargo, en ambos casos de $k_{\mathrm{h}}$ se observa que los empujes calculados numéricamente se incrementan al disminuir la frecuencia de la excitación (incremento en la razón $\lambda / H$ ). Otros investigadores han obtenido resultados similares (e.g. Gazetas et al., 2004), indicando que al aumentar la frecuencia de la excitación, los esfuerzos sísmicos ejercidos sobre elementos de retención disminuyen a valores incluso por debajo de los calculados mediante M-O. Por otra parte, analizando los empujes normalizados de la Figura $7 \mathrm{~b}$ en términos de los cocientes $F_{\text {sitio }}$ y $F / F_{0}$, se observa que para ambos casos de $k_{\mathrm{h}}$, los empujes calculados numéricamente se incrementan conforme la razón $F / F_{\text {sitio }}$ se acerca a la unidad. En esta comparación se destacan los casos $\mathrm{F} /$ $F_{\text {sitio }}=1.81$ y 2.17 , en los cuales se obtuvieron empujes numéricos mayores a los calculados con $\mathrm{M}-\mathrm{O}$, sugiriendo que la amplificación dinámica jugó un papel importante en los empujes desarrollados, aspecto imposible de tomar en cuenta mediante el método M-O. Este resultado coincide con lo obtenido por otros investigadores en análisis no lineales similares a los aquí presentados (Gazetas et al., 2004; Leuzzi et al., 2010). Por otro lado, la relación de los empujes con el cociente $F / F_{0}$ encuentra su valor mínimo para el caso $F / F_{0} \approx 1$, y pareciera aumentar al decrecer la razón $F / F_{0}$. Esto podría sugerir efectos de fase entre el muro y el suelo inmediatamente detrás. Para averiguar esta relación entre fases se podrían analizar las series de tiempo del muro y el suelo, aspecto que requiere mayor investigación y que no se abordará en este trabajo. Sin embargo, Athanasopoulos-Zekkos et al. (2013) realizaron una investigación sobre este aspecto y sus resultados sugieren que no existe una dependencia sistemática entre la razón $F / F_{0}$ y los empujes sísmicos desarrollados sobre muros cantiléver.

En la Figura 8 se muestran las distribuciones de esfuerzos dinámicos obtenidos para los casos $\lambda / H=5, \lambda / H=13 \mathrm{y}$ para todas las razones $\lambda H$. Se observa que para las altas frecuencias $(\lambda / H=5)$ las presiones aumentan en la parte superior del muro, mientras que para la menor frecuencia aplicada las presiones mayores tienden a concentrarse en torno al centro del muro. Estos resultados dan cuenta de los efectos de interacción entre el muro y el suelo en función de la frecuencia de la excitación, mostrando la importancia del contenido de frecuencia de la excitación. Resultados de otros investigadores (e.g. Cakir, 2013), muestran que el contenido de frecuencia de la excitación es uno de los parámetros más importantes a considerar en el análisis y diseño sísmico de elementos de retención, lo cual no se puede tomar en cuenta mediante métodos simplificados como M-O.

En la Figura 8c se presentan los valores medios (promediados en el tiempo de aplicación de la carga) de las distribuciones de presiones, donde se observa la variación en la respuesta del muro, acusando los efectos de interacción entre el relleno y la estructura. Se observa que los esfuerzos totales llegan a ser incluso mayores que los calculados para el caso $k_{\mathrm{h}}=$ PGA en la parte inferior del muro. Esto es congruente con el nivel de desplazamiento calculado, ya que en la parte inferior del muro se obtienen los menores desplazamientos (ver Figuras 6 y 10), lo cual se traduce en un aumento de los esfuerzos. Mientras que lo contrario se aplica para la parte superior del muro donde el suelo sufre las mayores deformaciones y por ende se concentran los efectos no lineales originando así una disminución de las presiones (mayor ductilidad en la parte superior). Estos resultados son congruentes con lo obtenido por Gazetas et al. (2004), quienes observaron que para muros flexibles (e.g., muros diafragma y pilas con anclajes), las presiones sísmicas en la mayoría de los casos son menores a las calculadas con $\mathrm{M}-\mathrm{O}$, especialmente en la mitad superior de las estructuras.
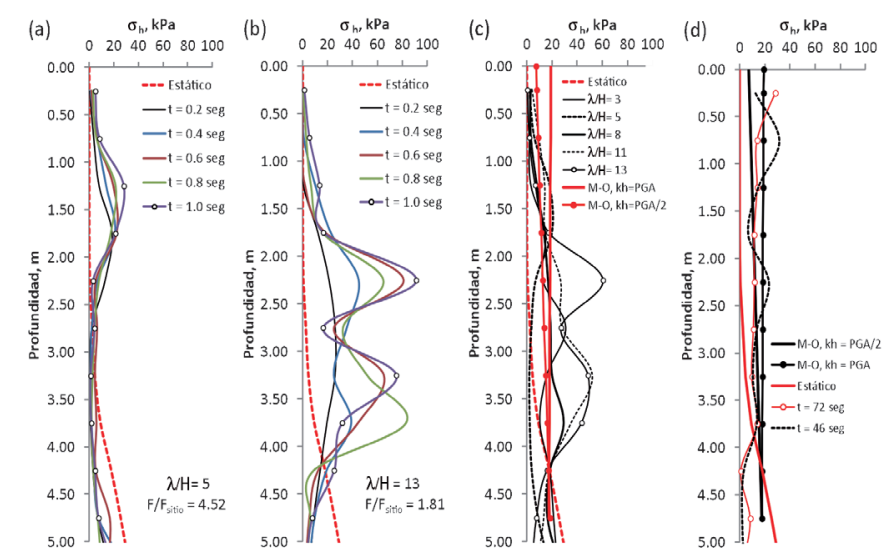

Figura 8: Esfuerzos dinámicos para a) $\lambda / H=5$, b) $\lambda / H=13$, c) todos los casos de $\lambda / H, y$ d) distribución de esfuerzo para carga sísmica correspondientes al instante indicado 


\section{Empujes bajo carga sísmica}

Los resultados se presentan en términos de espectros de respuesta, desplazamientos absolutos del suelo detrás del muro y variación de los esfuerzos dinámicos normalizados. En la Figura 9a se observa que el espectro de respuesta en el suelo detrás del muro $(Z=0)$ presenta ordenadas espectrales más bajas que el espectro de campo libre e incluso menores que el espectro a nivel de la base del muro $(Z=-5)$. Esto se atribuye a los efectos no lineales inducidos en el suelo por la carga sísmica. Por otro lado, se observa también que el espectro de campo libre es prácticamente idéntico al calculado con SHAKE. Esto indica que la respuesta de campo libre no se ve influenciada por efectos no lineales.

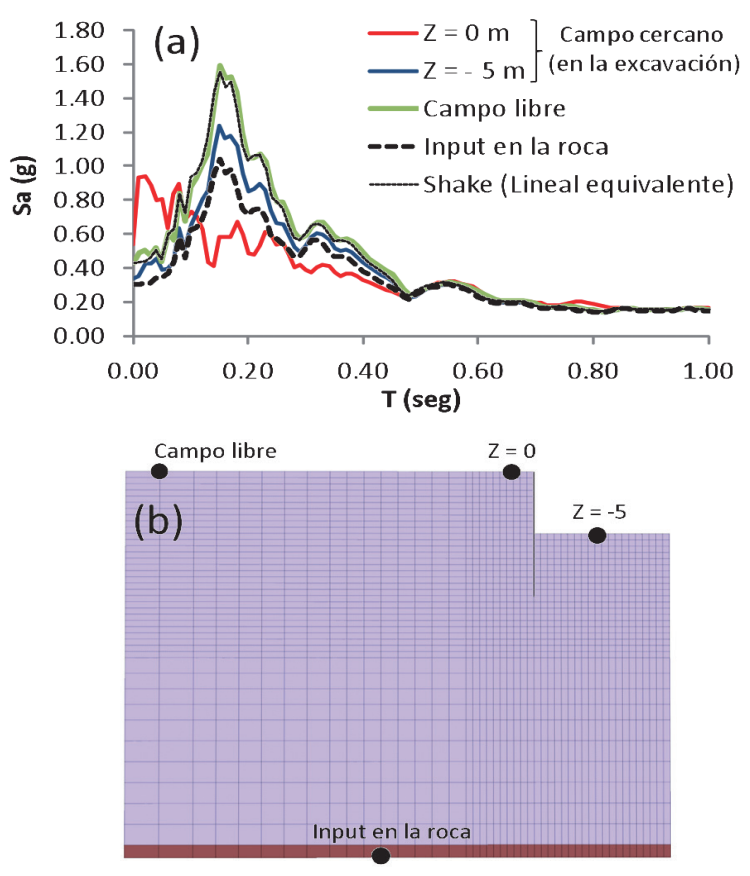

Figura 9: a) Espectros de respuesta y b) ubicación de puntos de monitoreo

En términos de los empujes, en la Figura 10b se muestra la variación en el tiempo de los empujes normalizados de dos formas distintas: empujes sísmicos (estáticos + dinámicos) normalizados por el empuje estático (serie Sigma/estático), y empujes sísmicos normalizados por el empuje calculado con el método de Mononobe-Okabe considerando dos valores distintos del coeficiente sísmico (series Sigma/ M-O). Se observa que, al contrario del caso de carga armónica, para el sismo utilizado, los empujes obtenidos son menores que los calculados con M-O. Esto es esperable ya que la carga armónica tiene una mayor intensidad (40
$\%$ más para el caso analizado, en términos de Intensidad de Arias). La diferencia observada pudiera atribuirse también a la interacción de las distintas ondas de diversas frecuencias actuando en forma simultánea, generando interferencias tanto constructivas como destructivas. Por otro lado, evidencia experimental para el caso de muros cantiléver, obtenidos por Kloukinas et al. (2013) mediante ensayos de mesa vibradora utilizando señales armónicas con frecuencias similares a las aquí analizadas (4, 7, 13, 25 y $43 \mathrm{~Hz}$ ), muestran que la respuesta dinámica del muro y el suelo son notoriamente distintas entre sí. Mientras que para el caso de carga sísmica, sus resultados mostraron que para los casos analizados por ellos, pueden obtenerse distribuciones de aceleración relativamente uniformes en el suelo, guardando cierta semejanza con la hipótesis pseudo estática. Sin embargo, para los casos en donde la amplificación dinámica sea importante, los métodos simplificados no necesariamente serán conservadores (Leuzzi et al., 2010). Una muestra de esto son los resultados para carga armónica aquí presentados. En la Figura 8d se observa también que la distribución en profundidad de los esfuerzos sísmicos sobre el muro presenta valores mayores a los calculados mediante $\mathrm{M}-\mathrm{O}$ en la parte superior del muro, lo cual debiera tomarse en cuenta para fines de diseño. Sería interesante comparar resultados en términos de distribución de momento flector para averiguar la influencia de la distribución de presiones con $\mathrm{M}-\mathrm{O}$ y las calculadas numéricamente.

Sería interesante investigar la variación de los empujes sísmicos para distintas razones $F / F_{\text {sitio }}$ considerando registros sísmicos reales $\left(F / F_{\text {sitio }}=0.44\right.$ considerando la frecuencia media del sismo calculada como un promedio ponderado de las amplitudes del espectro de Fourier), para averiguar en qué rangos el método M-O es conservador, ya que existen investigaciones que sugieren que para la mayoría de los casos, las presiones calculadas con M-O son conservadoras (Gazetas et al., 2004; Al-Atik y Sitar, 2010).

En cuanto a los movimientos del muro, en la Figura 10a pueden verse los desplazamientos totales en el punto $Z$ $=0$ para los casos de carga armónica y carga sísmica. El tiempo se encuentra normalizado por la duración de cada carga $T_{\mathrm{d}}$. Se observa que el caso de carga armónica induce los mayores desplazamientos como es de esperarse debido a su mayor severidad. 


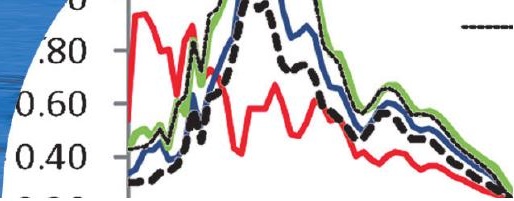

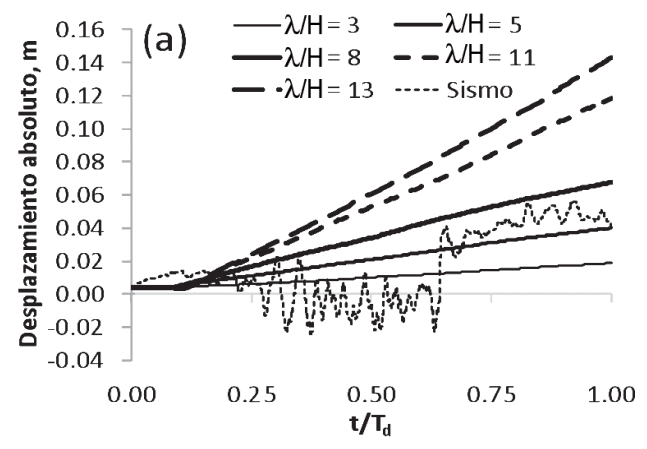

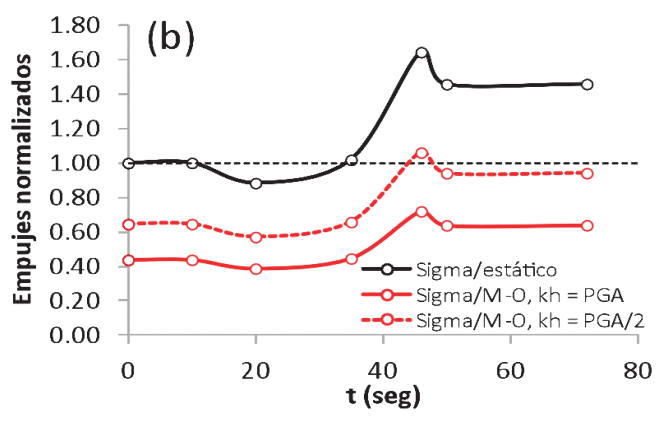

Figura 10: a) Desplazamientos absolutos en el punto $Z=0$ y b) empujes normalizados

\section{Conclusiones}

Para el caso de carga armónica se encontró que los empujes totales actuando en el muro observaron un valor mínimo para $\lambda H=5$, donde se obtuvo que los empujes calculados mediante Mononobe y Okabe M-O fueron mayores que los calculados numéricamente. Será interesante verificar en cálculos futuros si esta relación se conserva para otras geometrías de muros embebidos en diversas condiciones geotécnicas. De existir una relación de longitud de onda entre altura de muro que minimice las cargas sísmicas en muros de este tipo, sería de utilidad práctica determinarla para optimizar el comportamiento sísmico de este tipo de estructuras, por lo que el tema se abordará en trabajos futuros.

Al comparar resultados de empujes totales obtenidos con cargas armónicas y carga sísmica, se observó que esta última puede producir resultados más en línea con el método analítico de M-O. La influencia del contenido de frecuencia en los empujes dinámicos resulta evidente, lo cual es un hecho conocido en análisis dinámico. Será interesante verificar en qué rango de frecuencias el método M-O sigue siendo adecuado para el análisis de empujes dinámicos para determinar su rango de aplicación en términos de contenido de frecuencias. Como trabajo futuro se complementará la investigación utilizando como excitación registros sísmicos reales con distintos contenidos de frecuencias.

\section{Referencias}

Al-Atik, L. and N. Sitar (2010). Seismic earth pressures on cantilever retaining structures. Journal of Geotechnical and Geoenvironmental Engineering 136, No. 10, 1324- 1333

Athanasopoulos-Zekkos,A., Vlachakis, V.S. and Athanasopoulos, G.A. (2013). Phasing issues in the seismic response of yielding, gravity-type earth retaining walls - Overview and results from a FEM study. Soil Dynamics and Earthquake Engineering 55: $59-70$

Boroschek, R., Soto, P. and León, R. (2010). Maule region earthquake, February 27, 2010, Mw $=8.8$. RENADIC Report 10/08, Universidad de Chile

Cakir, T. (2013). Evaluation of the effect of earthquake frequency content on seismic behavior of cantilever retaining wall including soil-structure interaction. Soil Dynamics and Earthquake Engineering 45: 96-111

De la Hoz, K. (2007). Estimación de los parámetros de resistencia al corte en suelos granulares gruesos. Tesis de Grado, Universidad de Chile

Gazetas, G., Psarropoulos, P.N., Anastasopoulos, I. and Gerolymos, N. (2004). Seismic behaviour of flexible retaining systems subjected to short-duration moderately strong excitation. Soil Dynamics and Earthquake Engineering 24(7), 537-550

Idriss, I.M. and Sun, J.I. (1992). SHAKE91: a computer program for conducting equivalent linear seismic response analyses of horizontally layered soil deposits. User's Guide, University of California, Davis, 13 pp

Itasca (2009). FLAC3D. Fast Lagrangian Analysis of Continua in 3 Dimensions, Version 4.0. Itasca Consulting Group Inc., Minneapolis, MN, USA

Kloukinas, P. Santolo, A.S., Penna, A., Bhattacharya, S., Dietz, M., Dihoru, L., Evangelista, A., Simonellli, A.L., Taylor, C. and Mylonakis, G. (2013). Experimental investigation of dynamic behavior of cantilever retaining walls. $4^{\text {th }}$ ECCOMAS Thematic Conference on Computational Methods in Structural Dynamics and Earthquake Engineering, Kos island, Greece, 12-14 June 
Leuzzi, F., Foti, S., Lancellotta, R. and Mylonakis, G. (2010). Dynamic response of cantilever retaining walls considering soil non-linearity. $5^{\text {th }}$ International Conference on Recent Advances in Geotechnical Earthquake Engineering and Soil Dynamics, San Diego, California

Lysmer, J. and Kuhlemeyer, R.L. (1969). Finite dynamic model for infinite media. Journal of Engineering Mechanics 95(EM4), $859-877$

Méndez, B.C., Rivera, D. and Tastan, E.O. (2014). Static and dynamic soil pressures over a primary crusher station at a copper mine in Chile. Geo-Congress, 3198 - 3208, Atlanta, USA
Seed, H., Wong, T., Idriss, I.M. and Tokimatsu, K. (1986). Moduli and damping factors for dynamic analyses of cohesionless soils. Journal of Geotechnical Engineering 112(11): 1016-1032

SHAKE2000 (2008). A Computer Program for the ID Analysis of Geotechnical Earthquake Engineering Problems-User's Manual. GeoMotions, LLC, Lacey, USA 